\title{
Association network analysis identifies enzymatic components of gut microbiota that significantly differ between colorectal cancer patients and healthy controls
}

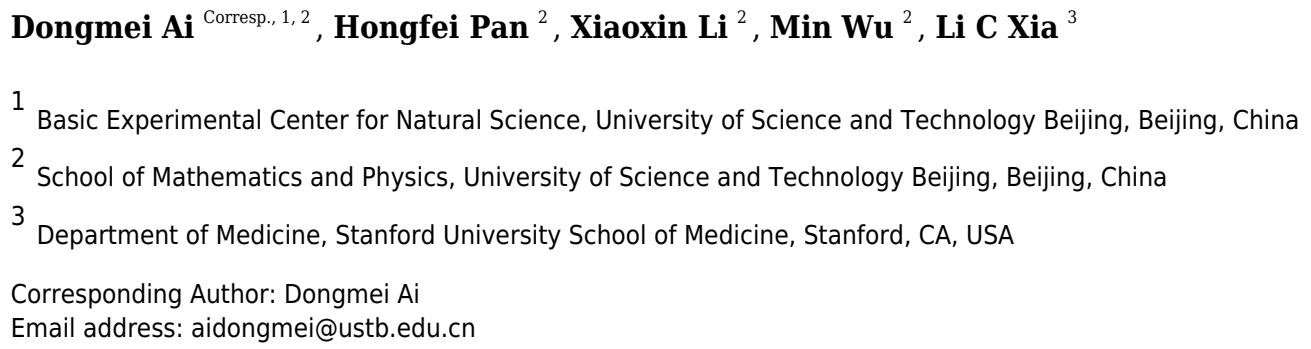

The human gut microbiota plays a major role in maintaining human health and was recently recognized as a promising target for disease prevention and treatment. Many diseases are traceable to microbiota dysbiosis, implicating altered gut microbial ecosystems, or, in many cases, disrupted microbial enzymes carrying out essential physiobiochemical reactions. Thus, the changes of essential microbial enzyme levels may predict human disorders. With the rapid development of high-throughput sequencing technologies, metagenomics analysis has emerged as an important method to explore the microbial communities in the human body, as well as their functionalities. In this study, we analyzed 156 gut metagenomics samples from patients with colorectal cancer (CRC) and adenoma, as well as that from healthy controls. We estimated the abundance of microbial enzymes using the HMP Unified Metabolic Analysis Network method (HUMAnN2) and identified the differentially abundant enzymes between CRCs and controls. We constructed enzymatic association networks using the extended local similarity analysis (ELSA) algorithm. We identified CRC-associated enzymic changes by analyzing the topological features of the enzymatic association networks, including the clustering coefficient, the betweenness centrality, and the closeness centrality of network nodes. The network topology of enzymatic association network exhibited difference between the healthy and the CRC environments. The ABC (ATP binding cassette) transporter and small subunit ribosomal protein S19 enzymes, had the highest clustering coefficient in the healthy enzymatic networks. In the contrast, the Adenosylhomocysteinase enzyme had the highest clustering coefficient in the CRC enzymatic networks. These enzymic and metabolic differences may serve as risk predictors for colorectal cancers and are worthy of further research. 


\section{Association network analysis identifies enzymatic}

2 components of gut microbiota that significantly differ

3 between colorectal cancer patients and healthy

\section{4 controls}

6 Dongmei $\mathrm{Ai}^{1,2^{*}}$, Hongfei $\mathrm{Pan}^{2}, \mathrm{Xiaoxin} \mathrm{Li}^{2}, \mathrm{Min} \mathrm{Wu}^{2}, \mathrm{Li}$ C. Xia ${ }^{3 *}$

$8{ }^{1}$ Basic Experimental Center for Natural Science, University of Science and Technology Beijing,

9 Xueyuan Road, Haidian District, Beijing, 100083, China

102 School of Mathematics and Physics, University of Science and Technology Beijing, Xueyuan

11 Road, Haidian District, Beijing, 100083, China

$12{ }^{3}$ Department of Medicine, Stanford University School of Medicine, 269 Campus Dr., Stanford,

13 CA 94305, USA

Corresponding Author:

Dongmei Ai

No.30, Xueyuan Road, Haidian District, 100083, Beijing, China

aidongmei@ustb.edu.cn

Li C. Xia

269 Campus Dr., Stanford, CA 94305, USA

21 1.c.xia@stanford.edu 


\section{Abstract}

The human gut microbiota plays a major role in maintaining human health and was recently recognized as a promising target for disease prevention and treatment. Many diseases are traceable to microbiota dysbiosis, implicating altered gut microbial ecosystems, or, in many cases, disrupted microbial enzymes carrying out essential physio-biochemical reactions. Thus, the changes of essential microbial enzyme levels may predict for human disorders. With the rapid development of high-throughput sequencing technologies, metagenomics analysis has emerged as an important method to explore the microbial communities in the human body, as well as their functionalities. In this study, we analyzed 156 gut metagenomics samples from patients with colorectal cancer (CRC) and adenoma, as well as that from healthy controls. We estimated the abundance of microbial enzymes using the HMP Unified Metabolic Analysis Network method (HUMAnN2) and identified the differentially abundant enzymes between CRCs and controls. We constructed enzymatic association networks using the extended local similarity analysis (ELSA) algorithm. We identified CRC-associated enzymic changes by analyzing the topological features of the enzymatic association networks, including the clustering coefficient, the betweenness centrality, and the closeness centrality of network nodes. The network topology of enzymatic association network exhibited difference between the healthy and the CRC environments. The ABC (ATP binding cassette) transporter and small subunit ribosomal protein S19 enzymes, had the highest clustering coefficient in the healthy enzymatic networks. In the contrast, the Adenosylhomocysteinase enzyme had the highest clustering coefficient in the CRC enzymatic networks. These enzymic and metabolic differences may serve as risk predictors for colorectal cancers and are worthy of further research. 
47 Introduction

48 The human body harbors hundreds of trillions of microorganisms (Huttenhower et al. 2012;

49 Turnbaugh et al. 2007). These microbial communities, comprising of bacteria, fungi, archaea, and

50 viruses, are highly complex. These microbes colonize internal and external surfaces, such as

51 mouth, esophagus, stomach, colon, respiratory tract, genitourinary tract and skin (Grice et al.

52 2009). In particular, the disruption of gut microbiota has been linked to a number of

gastrointestinal diseases (Cotter et al. 2013). With the rapid development of high-throughput

sequencing technologies, the metagenomics approach allowed us to extensively investigate the

human microbiota in their naturally occurring state. These findings will ultimately lead to a better

understanding of the gut microbiota in relation to the onset of gastrointestinal (GI) diseases (Sung

et al. 2016). The colonic microbiota also might promote colorectal cancer by eliciting host

responses (Cho \& Blaser 2012).

Gut microbiota was known to play an active role in gut homeostasis for many years. The gut microbial composition and metabolic activity can modify the host's susceptibility to diseases via homeostasis and lead to the onset of inflammation, dysplasia, and eventually cancer (Abreu \& Peek Jr 2014; Nistal et al. 2015). More recently, many specific species whose abundance were positively associated with CRC have been identified. Those included Streptococcus bovis, Helicobacter pylori, Bacteroides fragilis, Enterococcus faecalis, Clostridium septicum, Fusobacterium spp., and Escherichia coli (Boulangé et al. 2016; Gagnière et al. 2016).

However, these earlier works have primarily analyzed the species abundance and diversity differences among communities to reveal the associations between gut microbiota and CRC (Halfvarson et al. 2017; Sobhani et al. 2011). It was only recently, researchers begun to study microbes at the functional level. The development of metabolomics has markedly facilitated such endeavors. This is made possible because the microbial abundance change will disrupt microbial enzymes that carry out essential physio-biochemical reactions. Such enzymes, as profiled by metabolomics, thus may predict for human disorders. For examples, in the metabolic disorders, such as IBD or obesity, the changes in gut microbiota cause a state of imbalance in metabolic activity and lead to the diseases (Chang et al. 2015; Tsuda et al. 2010; Weir et al. 2013). 
microbial metabolic networks. Two primary methods are available to construct enzymatic networks: the constraint-based method (Borenstein 2012) and the topological theory-based method. The constraint-based method requires establishing a series of ecological constraint conditions, which requires iterative approximation of unknown parameters in turn. In contrast, the topological theory-based method constructs the network directly by pulling the annotated metabolic and biochemical reaction pathways from databases and removing redundant information from these pathway data as necessary.

Alternatively, in this paper, we demonstrated an approach to construct association networks by computing the local association coefficients of enzymes involved in biochemical reactions and constructing the enzymatic networks using these statistically significant local associations as edges and nodes. Using such derived enzymatic networks, we studied the differentially presented microbial enzymes and metabolic activities using topological analysis. Applying our approach to a large CRC dataset, we identified that the relative abundance of the enzymatic components of Bifidobacterium, Clostridium, Fusobacterium nucleatum, Porphyromonas gingivalis, and Eubacterium, were significantly higher in the adenoma and colorectal cancer patients, as compared to the healthy controls.

\section{Materials and methods}

\section{Cohort, fecal samples and metagenomic sequencing}

The metagenomic dataset (Zeller et al. 2014) used in this study consists of 156 fecal samples of randomly selected volunteers recruited from the Henri Mondor Hospital (Creteil, France), including 61 healthy people, 42 patients with colorectal adenoma, and 53 colorectal cancer patients. The dataset were downloaded from the EBI database, where the detailed data description can be found: https://www.ebi.ac.uk/ena/data/view/PRJEB6070.

\section{Estimation of enzyme abundance}

Raw metagenomics data were translated to functional enzymes using the Kyoto Encyclopedia of Genes and Genomes (KEGG) Ortholog database and the HMP Unified Metabolic Analysis Network 2 (HUMAnN2) software, which is a pipeline for efficient and accurate profiling of abundance in microbial pathways from a community based on metagenomic or metatranscriptomic sequencing data (Abubucker et al. 2012). We computed the difference of relative abundances for 2400 microbial enzymes involved in biochemical reactions between the CRC patients and the healthy controls. The results were standardized to eliminate potential bias in sequencing batches, and to remove any batch effects in enzyme abundances either specific to 
116

CRC patients or healthy controls.

HUMAnN2 uses an in-house script to normalize the abundance of enzymes. The script provides a method called 'total sum scaling' (TSS) normalization. The relative abundance is then calculated as follows:

$$
R \mathrm{a}_{i}=\frac{a_{i}}{\sum_{j} a_{j}}
$$

where $R \mathrm{a}_{i}$ is the relative abundance of the i-th enzyme and $a_{i}$ is its absolute abundance.

\section{Enzymatic association network construction}

We performed enzymatic association network construction using the Extended Local Similarity Analysis (ELSA) algorithm. ELSA was designed and developed by Xia et al. (2011) which uses a dynamic programming algorithm to effectively discover potential local and time-delayed associations of time-series and cross-sectional data. The dynamic programming algorithm can find all potential global, local and time-delayed associations between two series and identify the association with the highest similarity score as the maximum association between them. We used a false discovery rate Q-value cutoff of 0.05 to assess the statistical significance. The resulting enzymatic association networks representing statistically significant associations within the three environments were visualized by the Cytoscape (Shannon et al. 2003) software, in which nodes with blue borders in the three association networks represent enzymes enriched in the healthy controls, while yellow nodes represent enzymes enriched in colorectal cancer patients.

The ELSA algorithm has been extensively used in the association network analysis of microbial ecological data with a good performance (Weiss et al. 2016). Studies using ELSA have discovered symbiotic relationships among microbes and relationships between microbes and the environment that could not be identified by conventional correlation methods. For instance, Shade et al. found dynamic mixed associations between bacteria in lakes by using ELSA (Shade et al. 2010). Ki et al. examined the relationship between bacterial community structure and odor emissions during the degradation of soil and pig carcasses by using ELSA. This algorithm not only finds the associations between delayed data, but also effectively calculates the pairwise global associations between for multivariate series data (Ki et al. 2018).

\section{Network topological analysis}

Several topological measures were used in our network analysis, including the clustering 
148 coefficient and the betweenness and closeness centralities. The clustering coefficient is a

149 measure of the degree to which nodes in a graph tend to cluster together. The clustering

150 coefficient of the nodes represents their proximity in the network. An increased clustering

151 coefficient correlates with the higher tightness of the cluster involving the node and its

152 neighbors, hence, the node's importance. This feature of clustering coefficient allows its use for

153 the identification of key enzymes. The clustering coefficient is defined as

$$
C_{v}=\frac{2 n}{k(k-1)}
$$

where $\mathrm{n}$ is the number of edges between all the $\mathrm{k}$ neighbors of node $\mathrm{v}$.

\section{Statistical analysis}

We tested for the statistically significant abundance difference of enzymes associated with biochemical reactions of gut microbiota between the healthy controls, and adenoma or colorectal cancer patients by the two-tailed Wilcoxon rank-sum test. 
173

174

175

176

177

178

179

180

181

182

\section{Results and Discussion}

\section{Analysis of disease-associated enzymes}

We got 157 differentially abundant enzymes among healthy control, adenoma and CRC patients. We selected 13 enzymes with differentially node degree among healthy control, adenoma and CRC patients association network. In Figure 1, we identified 13 enzymes showing statistically significant changes in abundance levels. We conducted a detailed analysis of differential enzymes (IDs and names listed in Table 1) and associated microorganisms to better understand the relationships between microbial metabolic functions and human disease. The enzymes K02003 (ABC transport system ATP-binding protein, $\mathrm{P}=5.22 \mathrm{E}-04$ ), K06147 (ATP-binding cassette, subfamily $\mathrm{B}$, bacterial, $\mathrm{P}=9.80 \mathrm{E}-05$ ), K02025(Multiple sugar transport system permease protein, $\mathrm{P}=1.74 \mathrm{E}-06$ ), $\mathrm{K} 02965$ (Small subunit ribosomal protein $\mathrm{S} 19, \mathrm{P}=1.17 \mathrm{E}-04$ ), K02470(DNA gyrase subunit B,P=3.62E-04), K01624(Fructose-bisphosphate aldolase, $\mathrm{P}=$ 2.90E-04) , K02878 (Large subunit ribosomal protein L16, P=3.45E-04), K04043(Molecular chaperone DnaK, $\mathrm{P}=4$.66E-03), K09157(Uncharacterized protein, $\mathrm{P}=1.46 \mathrm{E}-05$ ), K01854(UDPgalactopyranose mutase, $\mathrm{P}=4.31 \mathrm{E}-06$ ), $\mathrm{K} 03091$ (RNA polymerase sporulation-specific sigma factor, $\mathrm{P}=2.10 \mathrm{E}-03$ ) were all significantly higher in healthy controls. The enzymes K01667(Tryptophanase, $\mathrm{P}=4.57 \mathrm{E}-04$ ) and K01251(Adenosylhomocysteinase, $\mathrm{P}=2.37 \mathrm{E}-03$ ) were all significantly higher in CRC patients. These difference trends were also observed between adenoma and CRC patients, however, with less significant P-values, suggesting that though adenoma is an intermediary stage in the CRC development, the enzymatic levels observed in adenoma patients resemble more the healthy controls' as compared to that of the CRC patients.

$\mathrm{K} 02003$ is the ABC (ATP binding cassette) transporter, and this protein showed a higher relative abundance in the healthy environment in comparison to either the adenoma or the colorectal environment. As one of the largest metabolic transport systems in humans, the ABC (ATP binding cassette) transporter system mainly transfers nutrients, biosynthetic precursors, trace metals, and vitamins, but it also transports lipids, drugs, and primary and secondary metabolites. It plays a major role in biosynthetic pathways. It was noted that low ATP-binding cassette protein subfamily (ABCB1,P-glycoprotein) protein levels may promote colorectal carcinogenesis (Andersen et al. 2013). ABCB1 protein levels were also found to be lower in CRC tissue as compared to welldifferentiated tissue (De Iudicibus et al. 2008).

In another example, K01854 (UDP-galactopyranose mutase (UGM), an enzyme that 
207

208

209

210

211

212

213

214

215

216

217

218

219

220

221

222

223

224

225

226

227

228

229

230

231

232

233

234

235

236

237

238

239

240

241

catalyzes chemical reactions) had a higher abundance in healthy samples compared to the other two groups (Figure 1). This enzyme is specifically involved in galactose metabolism, as well as amino sugar and nucleotide sugar metabolism, and it plays a major role in the production of cellular energy and the modification of proteins and glycolipids. In an early study, Petry et al. indicated the importance of galactose metabolism in humans. Galactose can be converted into energy. UGM is one of the main enzymes involved in galactose metabolism. Galactose metabolism is crucial for the health of both neonatal development and adults. Disruption of the production of this enzyme will affect galactose metabolism and even cause transferasedeficiency galactosemia in severe cases (Petry \& Reichardt 1998). Brown et al. have shown the presence of galactose metabolism disorders in colorectal cancer patients. Based on our metabolic association network, we have shown that the abundance of K01854 is lower in the colorectal cancer patients than the healthy controls, in agreement with previous findings (Brown et al. 2016).

We also found that UDP-galactopyranose mutase, a main enzyme involved in galactose metabolism, was more abundant in healthy people. The result again indicated that the reduced galactose metabolism was associated with the development of colorectal cancer. The main enzymatic active genera of galactose metabolism are Bacteroides spp. and Bifidobacterium spp.. The Bacteroides spp. participates in polysaccharide degradation and the Bifidobacterium spp. participates in galactose metabolism in the gut. Thus, both of these genera may exert protective effects against developing colorectal cancer.

There are enzymes had significantly higher abundances in cancer patients. The most prominent enzymes of the kind were K01251 (adenosylhomocysteinase) and K01667 (tryptophanase) (Figure 1). Therefore, the microbial species producing adenosylhomocysteinase, an enzyme involved in tryptophan metabolism, may facilitate tumor progression as it presented a significantly higher abundance in cancer patients. By using gas chromatography, Kim et al. found adenosylhomocysteinase to be a protein marker for colorectal cancer (Kim et al. 2009; Yin et al. 2013). Interestingly, our enzymatic association network also showed an enriched abundance of K01667 (tryptophanase) in colorectal cancer samples. Tryptophanase is a basic amino acid, and tryptophanase metabolism can help cancer escape immune surveillance. Indoleamine 2,3-dioxygenase 1 (IDO1) is a tryptophan-catabolizing enzyme and the main enzyme expressed in malignant inflamed gut. In addition, clinical data have shown that tryptophan metabolism promotes tumor progression (Santhanam et al. 2016). 
242

243

244

245

246

247

248

249

250

251

252

253

254

255

256

257

258

259

260

261

262

263

264

265

266

267

268

269

270

271

272

273

274

275

\section{Topological attributes of enzymatic association networks}

In Figure S1, we showed the global enzymatic association networks we constructed from the ELSA analysis of the enzymic abundance values. Meanwhile, we exhibited the enzymatic network topological attributes of thirteen enzymes in Table 2, including the clustering coefficient, and the closeness and betweenness centralities. As we can see, in the order from healthy, to adenoma and to colorectal cancer samples, there is a decreasing trend of node degree among the enzymes enriched in healthy controls (nodes with blue circles). Here, the degree of a node refers to the number of neighboring nodes that the node has. Such decreasing trend suggested that these enzymes were highly cooperating in the metabolic processes in health controls (Figure S1A), while the level of cooperation was reduced in adenoma (Figure S1B) and further reduced to minimum in the colorectal cancer environment (Figure S1C). Notably, a similar decreasing trend was observed in the clustering coefficient (Table 2) of healthy control enriched enzymes, which suggested a gradual loss of importance of these healthy enzymes in a pathogenic CRC gut microbial environment.

The enzyme K02003, an ABC (ATP binding cassette) transporter, had the highest clustering coefficients in the healthy enzymatic network. This ABC transporter was also identified as significantly more abundant in healthy samples by abundance level (see Figure 1). Both results consistently suggested its status as a signature enzyme species for the healthy gut microbial environment. Similarly, the clustering coefficient of enzyme K02965 (small subunit ribosomal protein S19) was one in the healthy network; however, the coefficient was zero in the colorectal cancer network, also suggesting that the enzyme has an important biochemical role in the healthy people, which was no longer found in cancer patients. increasing connectivity from healthy, to adenoma and to colorectal cancer enzymatic networks. Several such enzymes were uniquely presented in the CRC network. For instance, the K01251 enzyme (Adenosylhomocysteinase), which is a highly connected node in the CRC and adenoma network, however, did not show up in the healthy network at all (Figure 1), suggesting its functional role in the early stage of tumorigenesis. We also found that the betweenness centrality of K01251 was higher in the colorectal cancer environment, and zero in the healthy controls (see Table 2). These results suggest that K01251 may play a negative role in intestinal flora metabolism causing its enrichment in the colorectal cancer patients. 


\section{Conclusion}

278 We analyzed a large human gut metagenomics dataset by using the HUMAnN2 tool to estimate

279 the relative abundances of microbially produced enzymes in the healthy control, the adenoma

280 and the colorectal cancer patient samples, respectively. We identified differentially abundant

281 enzymes among these healthy, adenoma, and colorectal cancer patient groups. We constructed

282 enzymatic association networks representing the healthy, adenoma, and colorectal cancer

283 microbial environments using the ELSA algorithm, and analyzed the topological attributes of the

284 resulting networks.

The new enzymatic network analysis approach we took in this study addressed the issue in previous studies that the enzymatic association networks were constructed only for individual metabolic pathways involving a limited number of microorganisms. We were able to globally integrated thousands of enzymes and hundreds of metabolic substrates carrying out biochemical reactions. These microbial enzymatic networks represent the set of essential relationships between enzymes of microbial origin. These relationships were indicators of underlying biochemical reactions and production of metabolites. Among the methods available to study such relationships between microbes and metabolites, only this systematic network-based study can reveal these relationships between microbial communities and their host in high resolution.

The innovative point of our network construction is to use an extensively tested and validated metric - local similarity score for building edges in the network. We know various noises and biases are associated with large-scale network construction, which incur challenges in subsequent topological analysis. And many constructed enzymatic networks are not specific simply because of the presence of irrelevant metabolites. The use of an accurate and robust association metrics helped us in truthfully representing these metabolic interactions.

Moreover, the topological attributes of the enzymatic network can carry important information of underlying dynamics between the enzymes and the tumorigenesis process. For example, the clustering coefficient is a positive indicator of an enzyme's cooperativity with other enzymes. By systematically constructing the networks using the ELSA algorithm and datamining in node degree, clustering coefficient, betweenness and closeness centralities of the enzymatic network under different tutorial stages, we were able to identify signature enzymic species as well as the local shift in network structure of cooperating enzymes as potential cancer 
310

311

312

313

314

315

316

317

318

319

320

321

322

323

324

325

326

327

328

329

330

331

332

333

334

335

336

337

338

339

340

341

342

343

344

345

346

347

348

risk markers, which merit further research.

\section{Acknowledgements}

Dongmei Ai thanks Professor Fengzhu Sun at the University of Southern California. Li.C.Xia. thanks Dr. Nancy Zhang at the University of Pennsylvania and Dr. Hanlee Ji at Stanford University for their support and helpful discussions.

\section{References}

Abreu MT, and Peek Jr RM. 2014. Gastrointestinal malignancy and the microbiome. Gastroenterology 146:1534-1546. e1533.

Abubucker S, Segata N, Goll J, Schubert AM, Izard J, Cantarel BL, Rodriguez-Mueller B, Zucker J, Thiagarajan M, and Henrissat B. 2012. Metabolic reconstruction for metagenomic data and its application to the human microbiome. PLoS computational biology 8:e1002358.

Andersen V, Vogel U, Godiksen S, Frenzel FB, Sæbø M, Hamfjord J, Kure E, and Vogel LK. 2013. Low ABCB1 gene expression is an early event in colorectal carcinogenesis. PloS one 8:e72119.

Borenstein E. 2012. Computational systems biology and in silico modeling of the human microbiome. Briefings in bioinformatics 13:769-780.

Boulangé CL, Neves AL, Chilloux J, Nicholson JK, and Dumas M-E. 2016. Impact of the gut microbiota on inflammation, obesity, and metabolic disease. Genome medicine 8:42.

Brown DG, Rao S, Weir TL, O’Malia J, Bazan M, Brown RJ, and Ryan EP. 2016. Metabolomics and metabolic pathway networks from human colorectal cancers, adjacent mucosa, and stool. Cancer \& metabolism 4:11.

Chang C-H, Qiu J, O’Sullivan D, Buck MD, Noguchi T, Curtis JD, Chen Q, Gindin M, Gubin MM, and van der Windt GJ. 2015. Metabolic competition in the tumor microenvironment is a driver of cancer progression. Cell 162:1229-1241.

Cho I, and Blaser MJ. 2012. The human microbiome: at the interface of health and disease. Nature Reviews Genetics 13:260.

Cipe G, Idiz UO, Firat D, and Bektasoglu H. 2015. Relationship between intestinal microbiota and colorectal cancer. World J Gastrointest Oncol 7:233-240.

Cotter PD, Ross RP, and Hill C. 2013. Bacteriocins - a viable alternative to antibiotics? Nature Reviews Microbiology 11:95.

De Iudicibus S, De Pellegrin A, Stocco G, Bartoli F, Bussani R, and Decorti G. 2008. ABCB1 gene polymorphisms and expression of P-glycoprotein and long-term prognosis in colorectal cancer. Anticancer research 28:3921-3928.

Gagnière J, Raisch J, Veziant J, Barnich N, Bonnet R, Buc E, Bringer M-A, Pezet D, and Bonnet M. 2016. Gut microbiota imbalance and colorectal cancer. World journal of gastroenterology 22:501.

Grice EA, Kong HH, Conlan S, Deming CB, Davis J, Young AC, Bouffard GG, Blakesley RW, 
Murray PR, and Green ED. 2009. Topographical and temporal diversity of the human skin microbiome. science 324:1190-1192.

Halfvarson J, Brislawn CJ, Lamendella R, Vázquez-Baeza Y, Walters WA, Bramer LM, D'Amato M, Bonfiglio F, McDonald D, and Gonzalez A. 2017. Dynamics of the human gut microbiome in inflammatory bowel disease. Nature microbiology 2:17004.

Huttenhower C, Gevers D, Knight R, Abubucker S, Badger JH, Chinwalla AT, Creasy HH, Earl AM, FitzGerald MG, and Fulton RS. 2012. Structure, function and diversity of the healthy human microbiome. Nature 486:207.

Ki B-M, Ryu HW, and Cho K-S. 2018. Extended local similarity analysis (eLSA) reveals unique associations between bacterial community structure and odor emission during pig carcasses decomposition. Journal of Environmental Science and Health, Part A:1-10.

Kim H-J, Kang HJ, Lee H, Lee S-T, Yu M-H, Kim H, and Lee C. 2009. Identification of S100A8 and S100A9 as serological markers for colorectal cancer. Journal of proteome research 8:1368-1379.

Nistal E, Fernández-Fernández N, Vivas S, and Olcoz JL. 2015. Factors determining colorectal cancer: the role of the intestinal microbiota. Frontiers in oncology 5:220.

Petry KG, and Reichardt JK. 1998. The fundamental importance of human galactose metabolism: lessons from genetics and biochemistry. Trends in Genetics 14:98-102.

Santhanam S, Alvarado DM, and Ciorba MA. 2016. Therapeutic targeting of inflammation and tryptophan metabolism in colon and gastrointestinal cancer. Translational Research 167:67-79.

Shade A, Chiu CY, and McMahon K. 2010. Differential bacterial dynamics promote emergent community robustness to lake mixing: an epilimnion to hypolimnion transplant experiment. Environmental microbiology 12:455-466.

Shannon P, Markiel A, Ozier O, Baliga NS, Wang JT, Ramage D, Amin N, Schwikowski B, and Ideker T. 2003. Cytoscape: a software environment for integrated models of biomolecular interaction networks. Genome research 13:2498-2504.

Sobhani I, Tap J, Roudot-Thoraval F, Roperch JP, Letulle S, Langella P, Corthier G, Van Nhieu JT, and Furet JP. 2011. Microbial dysbiosis in colorectal cancer (CRC) patients. PloS one 6:e16393.

Sung J, Hale V, Merkel AC, Kim P-J, and Chia N. 2016. Metabolic modeling with Big Data and the gut microbiome. Applied \& translational genomics 10:10-15.

Tsuda H, Ochiai K, Suzuki N, and Otsuka K. 2010. Butyrate, a bacterial metabolite, induces apoptosis and autophagic cell death in gingival epithelial cells. Journal of periodontal research 45:626-634.

Turnbaugh PJ, Ley RE, Hamady M, Fraser-Liggett CM, Knight R, and Gordon JI. 2007. The human microbiome project. nature 449:804.

Weir TL, Manter DK, Sheflin AM, Barnett BA, Heuberger AL, and Ryan EP. 2013. Stool microbiome and metabolome differences between colorectal cancer patients and healthy adults. PloS one 8:e70803.

Weiss S, Van Treuren W, Lozupone C, Faust K, Friedman J, Deng Y, Xia LC, Xu ZZ, Ursell L, 
390

391

392

393

394

395

396

397

398

399

400

401

402 and Alm EJ. 2016. Correlation detection strategies in microbial data sets vary widely in sensitivity and precision. The ISME journal 10:1669.

Xia LC, Steele JA, Cram JA, Cardon ZG, Simmons SL, Vallino JJ, Fuhrman JA, and Sun F. 2011. Extended local similarity analysis (eLSA) of microbial community and other time series data with replicates. BMC systems biology: BioMed Central. p S15.

Yin H-R, Zhang L, Xie L-Q, Huang L-Y, Xu Y, Cai S-J, Yang P-Y, and Lu H-J. 2013. HyperplexMRM: a hybrid multiple reaction monitoring method using mTRAQ/iTRAQ labeling for multiplex absolute quantification of human colorectal cancer biomarker. Journal of proteome research 12:3912-3919.

Zeller G, Tap J, Voigt AY, Sunagawa S, Kultima JR, Costea PI, Amiot A, Böhm J, Brunetti F, and Habermann N. 2014. Potential of fecal microbiota for early - stage detection of colorectal cancer. Molecular systems biology 10:766. 
Figure 1

Enzymes with significant differences of relative abundance in healthy people, adenoma patients, and colorectal cancer patients.

Green bar is the relative abundance of enzymes of healthy people. Orange bar is the relative abundance of enzymes of adenoma patients. Red bar is the relative abundance of enzymes of colorectal cancer patients. $* * *$ represent $p<0.001, * *$ represent $p<0.01, *$ represent $p<0.05$.

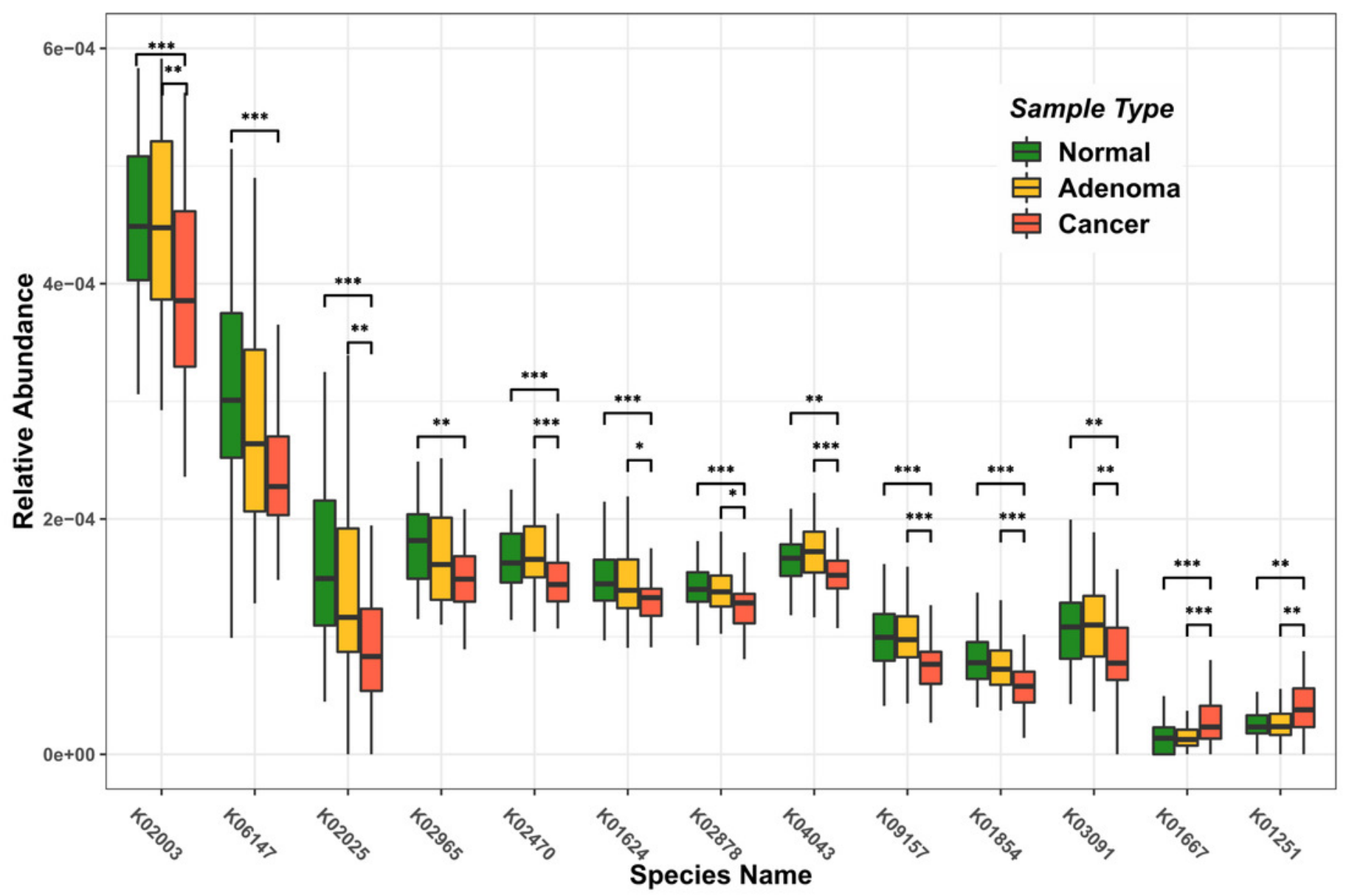


Table $\mathbf{1}$ (on next page)

Enzyme annotations 


\begin{tabular}{|l|l|}
\hline \multicolumn{1}{|c|}{ ID } & \multicolumn{1}{|c|}{ Name of Enzyme } \\
\hline K02003 & ABC transport system ATP-binding protein \\
\hline K06147 & ATP-binding cassette, subfamily B, bacterial \\
\hline K02025 & Multiple sugar transport system permease protein \\
\hline K02965 & Small subunit ribosomal protein S19 \\
\hline K02470 & DNA gyrase subunit B \\
\hline K01624 & Fructose-bisphosphate aldolase \\
\hline K02878 & Large subunit ribosomal protein L16 \\
\hline K04043 & Molecular chaperone DnaK \\
\hline K09157 & Uncharacterized protein \\
\hline K01854 & UDP-galactopyranose mutase \\
\hline K03091 & RNA polymerase sporulation-specific sigma factor \\
\hline K01667 & Tryptophanase \\
\hline K01251 & Adenosylhomocysteinase \\
\hline
\end{tabular}




\section{Table 2 (on next page)}

Topological attributes of 13 nodes of metabolic association networks in the healthy, adenoma, and colorectal cancer environments

The data range in the table is between $[0,1]$. 


\begin{tabular}{|c|c|c|c|c|c|c|c|c|c|}
\hline \multirow[b]{2}{*}{ ID } & \multicolumn{3}{|c|}{ Clustering Coefficient } & \multicolumn{3}{|c|}{ Closeness Centrality } & \multicolumn{3}{|c|}{ Betweenness Centrality } \\
\hline & Healthy & $\begin{array}{c}\text { Adenoma } \\
\text { of Gut }\end{array}$ & CRC & Healthy & $\begin{array}{c}\text { Adenoma } \\
\text { of Gut }\end{array}$ & CRC & Healthy & $\begin{array}{l}\text { Adenoma } \\
\text { of Gut }\end{array}$ & CRC \\
\hline K09157 & 7.42E-01 & $6.70 \mathrm{E}-01$ & 4.72E-01 & 5.66E-01 & 4.62E-01 & $4.14 \mathrm{E}-01$ & $8.76 \mathrm{E}-03$ & $4.58 \mathrm{E}-03$ & $4.62 \mathrm{E}-03$ \\
\hline K06147 & 7.79E-01 & 7.17E-01 & $5.49 \mathrm{E}-01$ & $6.09 \mathrm{E}-01$ & $6.10 \mathrm{E}-01$ & $5.03 \mathrm{E}-01$ & $3.75 \mathrm{E}-03$ & $5.71 \mathrm{E}-03$ & $8.28 \mathrm{E}-03$ \\
\hline K04043 & 7.81E-01 & 7.33E-01 & $5.00 \mathrm{E}-01$ & $5.72 \mathrm{E}-01$ & $5.12 \mathrm{E}-01$ & 4.17E-01 & $3.98 \mathrm{E}-03$ & $9.93 \mathrm{E}-03$ & $1.73 \mathrm{E}-03$ \\
\hline K03091 & $5.85 \mathrm{E}-01$ & $6.65 \mathrm{E}-01$ & 7.20E-01 & 4.97E-01 & $5.31 \mathrm{E}-01$ & 4.95E-01 & 4.32E-03 & 3.07E-03 & $6.63 \mathrm{E}-03$ \\
\hline K02965 & $1.00 \mathrm{E}+00$ & 7.56E-01 & $0.00 \mathrm{E}+00$ & 4.49E-01 & $4.38 \mathrm{E}-01$ & $3.00 \mathrm{E}-01$ & $0.00 \mathrm{E}+00$ & $7.65 \mathrm{E}-05$ & $0.00 \mathrm{E}+00$ \\
\hline K02878 & $9.62 \mathrm{E}-01$ & $6.98 \mathrm{E}-01$ & $0.00 \mathrm{E}+00$ & 4.61E-01 & $5.30 \mathrm{E}-01$ & $3.63 \mathrm{E}-01$ & $9.23 \mathrm{E}-06$ & $2.89 \mathrm{E}-03$ & $2.53 \mathrm{E}-04$ \\
\hline K02470 & 7.31E-01 & 7.18E-01 & $6.43 \mathrm{E}-01$ & $5.97 \mathrm{E}-01$ & $5.35 \mathrm{E}-01$ & 4.70E-01 & $8.81 \mathrm{E}-03$ & $3.79 \mathrm{E}-03$ & $3.20 \mathrm{E}-03$ \\
\hline K02025 & $8.17 \mathrm{E}-01$ & $6.12 \mathrm{E}-01$ & 4.83E-01 & $6.06 \mathrm{E}-01$ & $6.31 \mathrm{E}-01$ & $5.47 \mathrm{E}-01$ & $4.36 \mathrm{E}-03$ & $1.03 \mathrm{E}-02$ & $1.27 \mathrm{E}-02$ \\
\hline K02003 & $8.81 \mathrm{E}-01$ & 7.67E-01 & $8.02 \mathrm{E}-01$ & $5.37 \mathrm{E}-01$ & $5.67 \mathrm{E}-01$ & $4.52 \mathrm{E}-01$ & $1.20 \mathrm{E}-03$ & $3.68 \mathrm{E}-03$ & 7.40E-04 \\
\hline K01854 & $8.76 \mathrm{E}-01$ & $8.07 \mathrm{E}-01$ & $6.19 \mathrm{E}-01$ & $5.31 \mathrm{E}-01$ & $5.59 \mathrm{E}-01$ & 4.45E-01 & $2.72 \mathrm{E}-03$ & $1.65 \mathrm{E}-03$ & $1.84 \mathrm{E}-03$ \\
\hline K01667 & 7.33E-01 & $8.67 \mathrm{E}-01$ & $5.16 \mathrm{E}-01$ & $4.72 \mathrm{E}-01$ & $3.65 \mathrm{E}-01$ & 4.06E-01 & $2.82 \mathrm{E}-04$ & $5.77 \mathrm{E}-05$ & $3.82 \mathrm{E}-03$ \\
\hline K01624 & $9.82 \mathrm{E}-01$ & $7.93 \mathrm{E}-01$ & 7.33E-01 & $5.02 \mathrm{E}-01$ & $5.15 \mathrm{E}-01$ & $3.85 \mathrm{E}-01$ & $2.55 \mathrm{E}-05$ & $1.82 \mathrm{E}-03$ & 7.60E-04 \\
\hline K01251 & $0.00 \mathrm{E}+00$ & $0.00 \mathrm{E}+00$ & $5.90 \mathrm{E}-01$ & $0.00 \mathrm{E}+00$ & $4.38 \mathrm{E}-01$ & 4.03E-01 & $0.00 \mathrm{E}+00$ & $3.69 \mathrm{E}-04$ & $2.32 \mathrm{E}-03$ \\
\hline
\end{tabular}

